\title{
Factors associated with initial or subsequent choice of biologic disease- modifying antirheumatic drugs for treatment of rheumatoid arthritis
}

Yinzhu Jin ${ }^{1}$, Rishi J. Desai ${ }^{1}$, Jun Liu ${ }^{1}$, Nam-Kyong Choi ${ }^{1,2,3}$ and Seoyoung C. Kim ${ }^{1,4^{*}}$

\begin{abstract}
Background: Biologic disease-modifying antirheumatic drugs (DMARDs) are increasingly used for rheumatoid arthritis (RA) treatment. However, little is known based on contemporary data about the factors associated with DMARDs and patterns of use of biologic DMARDs for initial and subsequent RA treatment.

Methods: We conducted an observational cohort study using claims data from a commercial health plan (2004-2013) and Medicaid (2000-2010) in three study groups: patients with early untreated RA who were naïve to any type of DMARD and patients with prevalent RA with or without prior exposure to one biologic DMARD. Multivariable logistic regression models were used to examine the effect of patient demographics, clinical characteristics and healthcare utilization factors on the initial and subsequent choice of biologic DMARDs for RA.
\end{abstract}

Results: We identified a total of 195,433 RA patients including 78,667 (40\%) with early untreated RA and 93,534 (48\%) and 23,232 (12\%) with prevalent RA, without or with prior biologic DMARD treatment, respectively. Patients in the commercial insurance were $87 \%$ more likely to initiate a biologic DMARD versus patients in Medicaid $(O R=1.87,95 \%$ $\mathrm{Cl}=1.70-2.05)$. In Medicaid, African-Americans had lower odds of initiating $(\mathrm{OR}=0.59,95 \% \mathrm{Cl}=0.51-0.68$ in early untreated $\mathrm{RA} ; \mathrm{OR}=0.71,95 \% \mathrm{Cl}=0.61-0.74$ in prevalent $\mathrm{RA})$ and switching $(\mathrm{OR}=0.71,95 \% \mathrm{Cl}=0.55-0.90)$ biologic DMARDs than non-Hispanic whites. Prior use of steroid and non-biologic DMARDs predicted both biologic DMARD initiation and subsequent switching. Etanercept, adalimumab, and infliximab were the most commonly used first-line and second-line biologic DMARDS; patients on anakinra and golimumab were most likely to be switched to other biologic DMARDS.

Conclusions: Insurance type, race, and previous use of steroids and non-biologic DMARDs were strongly associated with initial or subsequent treatment with biologic DMARDs.

Keywords: Rheumatoid arthritis, Antirheumatic agents, Biologic therapy

\section{Background}

Rheumatoid arthritis (RA) is a common autoimmune inflammatory arthritis affecting over 1.3 million people in the USA [1]. Treatment with disease-modifying antirheumatic drugs (DMARDs) is considered the standard of care for RA [2,3]. The 2012 American College of

\footnotetext{
* Correspondence: skim62@partners.org

${ }^{1}$ Division of Pharmacoepidemiology and Pharmacoeconomics, Brigham and Women's Hospital, 1620 Tremont Street, Suite 3030, Boston, MA 02120, USA ${ }^{4}$ Division of Rheumatology, Immunology and Allergy, Brigham and Women's Hospital, Boston, MA, USA

Full list of author information is available at the end of the article
}

Rheumatology (ACR) guidelines recommend monotherapy with a non-biologic DMARD or double and triple therapy for early-stage RA [2]. Adding or switching to a tumor necrosis factor (TNF) inhibitor, abatacept, or rituximumab is recommended for patients who still experience moderate or high disease activity after 3 months of non-biologic DMARD treatment. If the initial biologic DMARD does not result in adequate response or causes adverse events, switching to another TNF inhibitor or non-TNF biologic drug is recommended [2]. 
Over the past two decades, major advances have occurred in the treatment of RA with development of novel biologic drugs targeting specific components of the immune system. A prior study based on a prospective RA cohort in California showed a remarkable change over the past two decades in the use of DMARDs in patients with longstanding RA. The proportion of patients receiving any biologic DMARD increased from 10 to 48\% between 1999 and 2009 [4]. The rising use of biologic drugs in RA in clinical practice as more drugs have become available supports a need for examining the characteristics of patients who receive biologic treatment and the relationship between the patients' characteristics and the biologic treatment. A number of biologic DMARDs are currently available for treatment of RA including five TNF inhibitors (adalimumab, certolizumab, etanercept, golimumab, and infliximab) and four nonTNF inhibitors (abatacept, anakinra, rituximab, and tocilizumab). Tofacitinib is an oral Janus kinase inhibitor for the treatment of RA, recently approved by the US Food and Drug Administration (FDA) in 2012 for treatment of RA with moderate to severe disease activity.

As recommended by ACR guidelines, the decision to start or switch a biologic DMARD should be made based on a patient's disease activity, prior DMARD treatment, and comorbid conditions [2]. Current evidence on predictors of biologic treatment in RA patients varies across studies. Some studies have demonstrated that initiation of biologic treatment is mainly associated with diseaserelated and treatment-related factors such as prior use of glucocorticoids, non-biologic DMARDs, or comorbidities $[5,6]$. Several studies have highlighted sociodemographic disparities in access to biologic DMARDs $[5,7,8]$. Older age was considered to be negatively associated with biologic treatment in all of these studies. These previous studies vary in terms of study period, RA disease stage, and population, leading to discrepancies in the conclusions. Furthermore, previous studies have only focused on initiation of biologic DMARDs and there is no evidence on switching biologic DMARDs as an outcome. In addition, little work has been done based on contemporary population-based data to describe the patterns of use of different biologic DMARDs for initial and subsequent RA treatment. Therefore, we conducted a cohort study using claims data from two large insurance programs from the USA to describe factors associated with initial and subsequent choice of biologic DMARD and the patterns of treatment sequence among various biologic DMARDs for the treatment of RA over 12 years.

\section{Methods}

\section{Data source}

We used the claims data from the US commercial health plan, United Healthcare (2004-2013), and a public health plan, Medicaid Analytic eXtract (MAX, 20002010). The United Healthcare plan insures primarily working adults and their family members across the USA, and Medicaid is a joint federal and state program that helps low-income individuals and families with the costs associated with medical and long-term custodial care. Both databases contain longitudinal information on pharmacy dispensing, medical diagnoses, procedures, hospitalizations, and physician visits across the USA. The study protocol was approved by the Institutional Review Board of the Brigham and Women's Hospital. Personal identifiers were removed from the dataset before the analysis to protect subject confidentiality. Patient informed consent was therefore not required.

\section{Study cohort}

We identified patients aged $\geq 18$ years with two diagnoses of RA (International Classification of Disease, Ninth Revision, ICD-9 diagnosis code 714.xx) that were $\geq 7$ days but $<365$ days apart. Eligible patients were required to have continuous insurance coverage between one year prior to the first RA diagnosis date and one year after the second RA diagnosis date. The second RA diagnosis date was defined as the index date, and the baseline period was defined as the time between the second RA diagnosis and one year prior to the first RA diagnosis (see Fig. 1). We

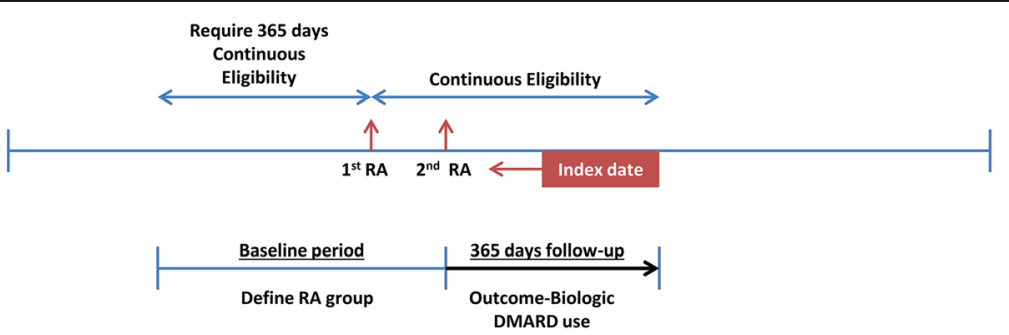

Fig. 1 Definition of the cohort. Eligible patients were required to have two diagnoses of rheumatoid arthritis (RA) that were $\geq 7$ days but $<365$ days apart, with continuous insurance coverage between one year prior to the first RA diagnosis $\left(1^{\text {st }} R A\right)$ date and one year after the second RA diagnosis $\left(2^{\text {nd }} R A\right)$ date. The second RA diagnosis date was defined as the index date, and the baseline period was defined as time between the second RA diagnosis and one year prior to the first RA diagnosis 
measured baseline DMARD use by identifying prescription fills of 21 individual non-biologic and biologic DMARDs. Non-biologic DMARDs were methotrexate (MTX), hydroxychloroquine (HCQ), sulfasalazine, leflunomide, gold compounds, mycophenolate mofetil, penicillamine, minocycline, azathioprine, cyclophosphamide, and cyclosporine. Biologic DMARDs included five TNF inhibitors (etanercept, adalimumab, certolizumab, golimumab, and infliximab), and four non-TNF biologic drugs (abatacept, anakinra, rituximab, and tocilizumab). We also included tofacitinib in the biologic group although this is a unique class of DMARD.

We separated the identified cohort into three mutually exclusive groups based on the stage of RA and use of biologic DMARDs during the baseline period. Group 1 was defined as patients with early untreated RA who had only one RA diagnosis in the baseline period and did not use any DMARDs during this time; group 2 was defined as patients with prevalent RA who were naive to biologic DMARDs but had used at least one non-biologic DMARD or had received more than one RA diagnosis in the baseline period; and group 3 was defined as patients with prevalent RA who had already used a single biologic DMARD during the baseline period.

\section{Study outcomes}

During the 12-month follow-up period, we identified initiation of the first-ever biologic DMARD among patients with early untreated RA, initiation of the first-ever biologic agent among biologic-DMARD-naïve patients with prevalent RA, and initiation of a second biologic agent among biologic-exposed patients with prevalent RA. The outcomes of the study were defined as binary variables (yes or no) indicating whether initiation or switching (initiation of a second biologic) of a biologic DMARD occurred.

\section{Predictors of interest}

Variables potentially related to biologic treatment decisions were assessed in the baseline period. First, we selected variables that indicate RA disease activity. These variables included prior use of steroids (groups 1-3), non-biologic DMARDs (groups 2-3), and biologic DMARDs for those already on a biologic agent (group 3). Underlying comorbid conditions and medication history that may affect choice of biologic treatment were chosen as potential predictors, which included hyperlipidemia, heart disease, hypertension, cerebrovascular accident, diabetes mellitus, obesity, psoriatic arthritis, ankylosing spondylitis, inflammatory bowel disease, history of hospitalization with severe infection, chronic obstructive pulmonary disease (COPD), liver disease, metastatic cancer, tumor, alcohol abuse, tobacco use, a combined comorbidity score [9], use of angiotensin- converting enzyme (ACE) inhibitors, angiotensin II receptor blockers (ARBs), beta blockers, calcium channel blockers, diuretics, insulin, oral hypoglycemic drugs, statins, non-statin lipid lowering drugs, aspirin, opioids, cyclooxygenase inhibitors (coxibs), and non-selective non-steroidal anti-inflammatory drugs (NSAIDs). Heart disease was defined as acute myocardial infarction, angina, chronic heart failure, or other forms of chronic ischemic heart disease. Liver disease was defined as chronic hepatitis, chronic liver disease and cirrhosis, other sequelae of chronic liver disease, or liver transplant. The combined comorbidity score is a single numerical score that combines 20 elements from the Charlson index and the Elixhauser system to summarize patients' co-morbid conditions [9]. We also included healthcare utilization factors to account for general patient health and contact with the healthcare system. These factors included the number of total distinct medications dispensed, number of physician visits, number of hospitalizations, and number of emergency room visits in the 1-year period prior to the index date. Finally, to identify predictors reflecting potential disparities in receipt of biologic DMARD treatment, we selected the insurance program (United Healthcare or Medicaid), age, gender, calendar year, region, and race/ethnicity (only available in Medicaid).

\section{Statistical analysis}

To estimate association between potential predictors and biologic initiation/switch, we applied multivariable logistic regression in each of the three cohort groups. Baseline covariates that we identified were all included in each model. In addition, in the models in the prevalent RA groups (groups 2 and 3) we also included the use of MTX, HCQ, and total number of different non-biologic DMARD prescriptions during the prior year. For group 3 we additionally included prior use of biologic DMARDs categorized by their generic names.

As we had information on patients' race and ethnicity only in the Medicaid database, we conducted an additional analysis to examine the racial/ethnicity disparities in biologic DMARD use. Some Medicaid patients also would be eligible for the Medicare plan (i.e., dualeligible patients). Since these patients may have different coverage for biologic agents than those with only Medicaid, we conducted sensitivity analysis by excluding Medicaid patients who also received Medicare coverage at any time during the entire study period. We applied the same aforementioned multivariable logistic model for this sensitivity analysis in each of the three different RA groups. All analyses were performed using SAS version 9.4. 


\section{Results}

Baseline characteristics

A total of 195,433 patients were identified: 82,402 (42.2\%) from the United Healthcare and 113,031(57.8\%) from the Medicaid database. The mean age was $49( \pm 12)$ years and 79\% were female. There were 78,667 patients with early untreated RA who did not have DMARD treatment before the index date. Among patients with prevalent RA there were 93,534 and 23,232 without or with prior biologic DMARD treatment, respectively (Table 1). Of the patients with early untreated RA, 66\% were enrolled in Medicaid, whereas $67 \%$ of patients with prevalent RA who already initiated biologic DMARDs were enrolled in the commercial insurance program.

The prevalence of comorbidities such as hyperlipidemia, hypertension, diabetes, and COPD was high (21-39\%) across all cohort groups: $43 \%$ of the overall cohort had used steroids prior to their initiation of DMARD treatment, and more than $60 \%$ of patients with prevalent RA had used steroids before initiating a biologic DMARD. The average daily steroid dose was $<5 \mathrm{mg} /$ day in most patients who had steroids during the baseline period. The proportion of medium (5-10 mg/day) and high ( $\geq 10 \mathrm{mg} /$ day) intake of steroids was higher in patients with prevalent RA than in patients with early untreated RA.

More than $70 \%$ of the patients with prevalent RA (both biologic-DMARD-naïve and prevalent users) had one non-biologic agent during their baseline period. One fifth of each of these two cohorts used more than one type of non-biologic DMARDs. Use of MTX, the most commonly used non-biologic DMARD, was common among both biologic-DMARD-naive patients with prevalent RA and those who had used a biologic DMARD before $(40.8 \%$ and $53.3 \%$, respectively, Table 1$)$.

\section{Factors associated with biologic initiation and subsequent} use

The multivariable logistic regression model (Table 2) showed strong association between the insurance type and the initiation of biologic DMARDs. The adjusted odds ratio (OR) of biologic DMARD initiation was 1.87 $(95 \% \mathrm{CI}=1.70-2.05)$ in patients who were enrolled in the United Healthcare program compared to patients under Medicaid coverage. The sensitivity analysis after excluding patients with dual eligibility yielded very similar results to those from the main analysis (results not shown). Older age was associated with decreased odds of biologic DMARD prescription in all three cohorts. With a 10-year increase in age, the likelihood of initiating a biologic DMARD reduced by $13 \%$ in patients with early untreated RA and by $29 \%$ in those with prevalent RA. The odds of switching to a different biologic DMARD were also decreased by $13 \%$ per 10 -year increase in age among patients with prevalent RA. Female patients were more likely to initiate or switch to a biologic DMARD than male patients.

In the Medicaid cohort, we observed strong race disparities in biologic DMARD utilization. After adjusting for demographics, comorbidities, medication history, and health utilization characteristics, African-Americans were $30-40 \%$ less likely to initiate biologic DMARDs $(\mathrm{OR}=0.59,95 \% \mathrm{CI}=0.51-0.68$ in the early untreated RA group; $\mathrm{OR}=0.71,95 \% \mathrm{CI}=0.61-0.74$ in the prevalent RA group) or to switch biologic DMARDs in the prevalent $\mathrm{RA}$ group $(\mathrm{OR}=0.71,95 \% \mathrm{CI}=0.55-$ $0.90)$ compared to the white, non-Hispanic population (Table 3).

We did not find associations between most of the comorbid conditions and initiating or switching biologic DMARDs. We observed decreased odds of biologic DMARDs initiation in patients who had early untreated $\mathrm{RA}$ and hypertension ( $\mathrm{OR}=0.85,95 \% \mathrm{CI}=0.78-0.94)$. Having inflammatory bowel disease increased the odds of initiation of biologic DMARDs but decreased that of switching. Alcohol abuse decreased the likelihood of biologic DMARD initiation ( $\mathrm{OR}=0.77,95 \% \mathrm{CI}=0.64-0.93$ ) in patients with early untreated RA. However, it was not associated with biologic DMARD initiation or switching among patients with prevalent RA.

Use of coxibs and non-selective NSAIDs was significantly associated with biologic initiation among early untreated and patients with prevalent RA. Patients with early untreated RA with prior use of Cyclooxygenase inhibitors (Coxibs) were 30-46\% more likely to initiate $(\mathrm{OR}=1.46,95 \% \mathrm{CI}=1.32-1.61)$ or switch biologic DMARDs (OR $=1.30,95 \% \mathrm{CI}=0.13-1.38)$. Similarly, patients with early untreated RA with prior use of NSAIDs were $30 \%$ more likely to initiate a biologic DMARD (OR $=1.3095 \% \mathrm{CI}=1.20-1.39$ ). Opioid use was associated with both biologic DMARD initiation and switching in all three cohorts. Steroid daily dosage was strongly associated with both biologic DMARD initiation and switching. In patients with early untreated RA, use of 5$10 \mathrm{mg} /$ day steroids on average increased the odds of initiating a biologic DMARD by $>200 \%$ (OR $=3.12$, 95\% $\mathrm{CI}=2.56,3.80)$ compared to no use. Similarly, in patients with prevalent RA, medium steroid daily dosage (5$10 \mathrm{mg} /$ day) also increased the odds of biologic DMARD initiation by almost $90 \%(\mathrm{OR}=1.89,95 \% \mathrm{CI}=1.76,2.04)$.

Patients with prevalent RA were more likely to initiate a biologic DMARD or switch to another biologic drug if they used more than one type of non-biologic drug during the baseline period $(\mathrm{OR}=2.40,95 \% \mathrm{CI}=2.17-2.67$ and $\mathrm{OR}=1.98,95 \% \mathrm{CI}=1.65-2.36$, respectively, Table 2 ). Prior use of MTX was associated with increased biologic DMARD initiation $(\mathrm{OR}=1.79,95 \% \mathrm{CI}=1.69-1.90)$ in patients with prevalent RA, whereas prior use of HCQ was associated with decreased biologic DMARD initiation 
Table 1 Baseline characteristics of the three cohorts

\begin{tabular}{|c|c|c|c|c|}
\hline & All & Early untreated RA & $\begin{array}{l}\text { Prevalent RA, naïve to } \\
\text { biologic DMARDs }\end{array}$ & $\begin{array}{l}\text { Prevalent RA, exposed } \\
\text { to biologic DMARDs }\end{array}$ \\
\hline Total number & $195,433(100)$ & $78,667(100)$ & $93,534(100)$ & $23,232(100)$ \\
\hline \multicolumn{5}{|l|}{ Insurance type } \\
\hline Commercial (United) & $82,402(42.2)$ & $26,810(34.1)$ & $40,023(42.8)$ & $15,569(67.0)$ \\
\hline Public (Medicaid) & $113,031(57.8)$ & $51,857(65.9)$ & $53,511(57.2)$ & $7663(33.0)$ \\
\hline Age & $49( \pm 12.1)$ & $49( \pm 12.2)$ & $50( \pm 12.1)$ & $49( \pm 11.9)$ \\
\hline Gender (female) & $153,933(78.8)$ & $60,487(76.9)$ & $75,456(80.7)$ & $17,990(77.4)$ \\
\hline \multicolumn{5}{|l|}{ Comorbid conditions } \\
\hline Hyperlipidemia & $76,793(39.3)$ & $36,538(46.4)$ & $33,005(35.3)$ & $7250(31.2)$ \\
\hline Heart disease ${ }^{a}$ & $34,869(17.8)$ & $17,501(22.2)$ & $14,896(15.9)$ & $2472(10.6)$ \\
\hline Hypertension & $92,080(47.1)$ & $41,338(52.5)$ & $42,244(45.2)$ & 8498 (36.6) \\
\hline Cerebrovascular accident & $10,118(5.2)$ & $5432(6.9)$ & $4175(4.5)$ & $511(2.2)$ \\
\hline Diabetes & $41,704(21.3)$ & $20,195(25.7)$ & $18,143(19.4)$ & $3366(14.5)$ \\
\hline Obesity & $22,828(11.7)$ & $12,317(15.7)$ & $8829(9.4)$ & $1682(7.2)$ \\
\hline Inflammatory bowel disease & $4037(2.1)$ & $1475(1.9)$ & $1661(1.8)$ & $901(3.9)$ \\
\hline History of hospitalization with severe infections ${ }^{b}$ & $8876(4.6)$ & $4539(5.8)$ & $3689(3.9)$ & $648(2.8)$ \\
\hline COPD & $62,388(31.9)$ & $30,789(39.1)$ & $26,974(28.8)$ & $4625(19.9)$ \\
\hline Liver disease & $13,030(6.7)$ & $7035(8.9)$ & $5057(5.4)$ & $938(4.0)$ \\
\hline Metastatic cancer & $1849(1.0)$ & $1028(1.3)$ & $719(0.8)$ & $102(0.4)$ \\
\hline Any tumor & $12,976(6.6)$ & $6301(8.0)$ & $5683(6.1)$ & $992(4.3)$ \\
\hline Alcohol abuse & $8699(4.5)$ & $5385(6.8)$ & $2928(3.1)$ & $386(1.7)$ \\
\hline Tobacco use & $35,992(18.4)$ & $18,567(23.6)$ & $14,562(15.6)$ & $2863(12.3)$ \\
\hline Combined comorbidity score & $1( \pm 2.0)$ & $2( \pm 2.0)$ & $1( \pm 1.9)$ & $1( \pm 1.5)$ \\
\hline \multicolumn{5}{|l|}{ History of medication use } \\
\hline ACE inhibitors & $35,742(18.3)$ & $15,088(19.2)$ & $17,107(18.3)$ & $3547(15.3)$ \\
\hline ARBs & $16,311(8.4)$ & $7159(9.1)$ & $7310(7.8)$ & $1842(7.9)$ \\
\hline Beta blockers & $30,934(15.8)$ & $12,955(16.5)$ & $14,679(15.7)$ & $3300(14.2)$ \\
\hline Calcium channel blockers & $28,302(14.5)$ & $11,936(15.2)$ & $13,820(14.8)$ & $2546(11.0)$ \\
\hline Diuretics & $52,688(27.0)$ & $22,093(28.1)$ & $25,279(27.0)$ & $5316(22.9)$ \\
\hline Insulin & $8851(4.5)$ & $4100(5.2)$ & $3920(4.2)$ & $831(3.6)$ \\
\hline Oral hypoglycemic & $21,004(10.8)$ & $95,28(12.1)$ & $9620(10.3)$ & $1856(8.0)$ \\
\hline Statins & $38,246(19.6)$ & $173,91(22.1)$ & $17,079(18.3)$ & $3776(16.3)$ \\
\hline Non-statin lipid-lowering drugs & $11,324(5.8)$ & $5213(6.6)$ & $4845(5.2)$ & $1266(5.4)$ \\
\hline Aspirins & $10,876(5.6)$ & $58,42(7.4)$ & $4517(4.8)$ & $517(2.2)$ \\
\hline COX-2 inhibitors & $44,555(22.8)$ & $150,28(19.1)$ & $24,130(25.8)$ & $5397(23.2)$ \\
\hline Non-selective NSAIDs & $108,196(55.4)$ & $468,58(59.6)$ & $51,080(54.6)$ & $10,258(44.2)$ \\
\hline Opioids & $118,542(60.7)$ & $485,45(61.7)$ & $56,545(60.5)$ & $13,452(57.9)$ \\
\hline Steroids & $106,676(54.6)$ & $33,474(42.6)$ & $57,604(61.6)$ & $15,598(67.1)$ \\
\hline Cumulative steroid dose (mg/day in prior year) & $3( \pm 32.8)$ & $2( \pm 3.2)$ & $4( \pm 43.3)$ & $4( \pm 20.3)$ \\
\hline \multicolumn{5}{|l|}{ Average daily steroid dose } \\
\hline None & $109,365(56.0)$ & $53,489(68.0)$ & $45,373(48.5)$ & $10,503(45.2)$ \\
\hline Low (<5 mg/day) & $66,779(34.2)$ & $22,933(29.2)$ & $35,157(37.6)$ & $8689(37.4)$ \\
\hline Medium (5-10 mg/day) & $12,610(6.5)$ & $1473(1.9)$ & $8408(9.0)$ & $2729(11.7)$ \\
\hline High ( $\geq 10$ mg/day) & $6679(3.4)$ & $772(1.0)$ & $4596(4.9)$ & $1311(5.6)$ \\
\hline
\end{tabular}


Table 1 Baseline characteristics of the three cohorts (Continued)

\begin{tabular}{|c|c|c|c|c|}
\hline Non-biologic drugs & - & - & $66,961(71.6)$ & $16,550(71.2)$ \\
\hline Number of non-biologic drugs & - & & & \\
\hline None & - & - & $26,573(28.4)$ & $6682(28.8)$ \\
\hline One & - & - & $48,124(51.5)$ & $11,822(50.9)$ \\
\hline More than one & - & - & $18,837(20.1)$ & $4728(20.4)$ \\
\hline Prior MTX & - & - & $38,172(40.8)$ & $12,381(53.3)$ \\
\hline Prior HCQ & - & - & $28,901(30.9)$ & $3735(16.1)$ \\
\hline \multicolumn{5}{|l|}{ Health care utilization } \\
\hline Number of prescriptions & $14( \pm 9.6)$ & $14( \pm 10.2)$ & $14( \pm 9.3)$ & $13( \pm 8.2)$ \\
\hline Number of physician visits & $11( \pm 9.4)$ & $11( \pm 9.5)$ & $11( \pm 9.4)$ & $12( \pm 8.4)$ \\
\hline Number of hospitalizations & $0( \pm 1.0)$ & $0( \pm 1.2)$ & $0( \pm 1.0)$ & $0( \pm 0.7)$ \\
\hline Number of emergency room visits & $1( \pm 3.0)$ & $1( \pm 3.6)$ & $1( \pm 2.7)$ & $1( \pm 1.7)$ \\
\hline
\end{tabular}

Values are the number (\%) or mean $( \pm \mathrm{SD})$. RA rheumatoid arthritis, DMARD disease-modifying antirheumatic drug, COPD chronic obstructive pulmonary disease, $A C E$ angiotensin-converting enzyme, ARB angiotensin II receptor blocker, COX cyclooxygenase, NSAID non-selective non-steroidal anti-inflammatory drug, MTX methotrexate, HCQ hydroxychloroquine. ${ }^{a}$ Acute myocardial infarction, angina, chronic heart failure, and other forms of chronic heart disease. ${ }^{b}$ History of hospitalization with serious bacterial infections or opportunistic infections

$(\mathrm{OR}=0.57,95 \% \mathrm{CI}=0.54-0.61)$. However, both MTX and HCQ indicated decreased odds of switching to another biologic DMARD among prevalent biologic DMARD users $(\mathrm{OR}=0.78$ and 0.75 , respectively).

\section{Patterns of switching biologic DMARDs}

Etanercept was the most commonly used (51\%) biologic drug during the baseline period, followed by adalimumab (22\%) and infliximab (22\%) (Table 4). Overall, 12\% of biologic DMARD users switched to other biologic DMARDs; specifically, the largest proportion of patients switching (32.7\%) among all the biologic agents was for anakinra, followed by golimumab (26.9\%), with adjusted OR of $3.20(95 \% \mathrm{CI}=2.41-4.25)$ and $2.24(95 \%$ $\mathrm{CI}=1.48-3.37$ ), respectively (Table 4). Overall, over two thirds of patients switched from a TNF/non-TNF inhibitor to another TNF inhibitor.

\section{Discussion}

Our study presents a comprehensive list of factors associated with biologic initiation or switching, and patterns of biologic DMARD use in patients with RA, based on large longitudinal databases representing both private and public sectors over the 12-year study period. We identified that initiation or switching of biologic DMARDs was associated with factors that are suggestive of history of RA treatment, such as previous use of steroids and non-biologic DMARDs. In addition, we noted that there are significant disparities in receipt of biologic treatment, by race and insurance type. For patterns of initial and subsequent choice of biologic treatment, we observed that TNF inhibitors including etanercept, adalimumab, and infliximab were the most widely used firstline and second-line biologic DMARDs, and patients were more likely to switch from golimumab or anakinra to a TNF inhibitor.

Our results showed lower odds of initiating a biologic DMARD among patients covered by public sector insurance compared to those covered by private insurance. This suggests different accessibility to biologic DMARDs under these two types of insurance programs. Fischer et al. reported that 32 out of 50 states studied had implemented or planned to implement prior authorization policies for biologic DMARDs, which may be a barrier to accessing them [10]. However, the type of health care insurance was only associated with the initial utilization of biologic DMARDs. Once RA patients had been initiated on biologic DMARDs, the insurance type did not seem to be associated with subsequent switching to other biologic DMARDs $(\mathrm{OR}=0.92,95 \% \mathrm{CI}=0.81-1.05)$.

We also found strong racial disparities in biologic DMARD utilization. Initiation of a biologic DMARD in patients with early untreated RA and in patients with prevalent RA was $40 \%$ and $30 \%$ less likely in the African-American population compared to the white population. A similar pattern was observed in previous observational studies [11-13]. We also observed that subsequent switch to another biologic DMARD in African-Americans was 30\% less likely than in the white population. Disparities in biologic DMARD switching have not been reported elsewhere. Solomon et al. demonstrated that a visit to the rheumatologist acted as a mediator and after adjusting for rheumatology visit the effect of African-American race in any DMARD use was attenuated [11]. We were not able to adjust for rheumatology visit, since information on provider specialty is not available in the Medicaid database. But after adjusting for insurance type and other healthcare utilization 
Table 2 Adjusted odds ratio (95\% CI) of initiation or switching biologic DMARDs

\begin{tabular}{|c|c|c|c|}
\hline & $\begin{array}{l}\text { Biologic DMARD initiation among } \\
\text { patients with early untreated RA }\end{array}$ & $\begin{array}{l}\text { Biologic DMARD initiation among } \\
\text { patients with prevalent RA }\end{array}$ & $\begin{array}{l}\text { Switching of biologic DMARDs } \\
\text { among biologic DMARD users }\end{array}$ \\
\hline Total number & $78,667(100 \%)$ & $93,534(100 \%)$ & $23,232(100 \%)$ \\
\hline Biologic DMARD initiation/switch, $n$ & $3873(4.9 \%)$ & $10,361(11.1 \%)$ & $2761(11.9 \%)$ \\
\hline \multicolumn{4}{|l|}{ Data source } \\
\hline United vs. Medicaid & $1.87(1.70,2.05)$ & $1.13(1.06,1.20)$ & $0.92(0.81,1.05)$ \\
\hline Age (by 10-year increase) & $0.87(0.84,0.89)$ & $0.81(0.79,0.83)$ & $0.87(0.84,0.91)$ \\
\hline \multicolumn{4}{|l|}{ Gender } \\
\hline Male vs. female & $0.90(0.83,0.98)$ & $0.95(0.89,1.00)$ & $0.87(0.79,0.97)$ \\
\hline \multicolumn{4}{|l|}{ Comorbid conditions in prior year } \\
\hline Hyperlipidemia & $0.90(0.83,0.97)$ & $0.97(0.92,1.03)$ & $1.02(0.91,1.13)$ \\
\hline Heart disease & $0.92(0.82,1.03)$ & $1.02(0.94,1.10)$ & $1.12(0.96,1.31)$ \\
\hline Hypertension & $0.85(0.78,0.94)$ & $0.91(0.86,0.97)$ & $0.94(0.83,1.06)$ \\
\hline Cerebrovascular accident & $0.91(0.76,1.08)$ & $0.94(0.83,1.06)$ & $1.21(0.92,1.57)$ \\
\hline Diabetes mellitus & $0.93(0.83,1.04)$ & $1.01(0.93,1.09)$ & $0.95(0.80,1.12)$ \\
\hline Obesity & $1.02(0.92,1.12)$ & $0.98(0.91,1.06)$ & $0.99(0.85,1.16)$ \\
\hline Inflammatory bowel disease & $1.89(1.56,2.30)$ & $1.22(1.06,1.42)$ & $0.71(0.56,0.89)$ \\
\hline Hospitalization with severe infection & $0.82(0.67,1.00)$ & $1.03(0.91,1.16)$ & $0.86(0.66,1.11)$ \\
\hline COPD & $0.80(0.74,0.87)$ & $0.97(0.92,1.02)$ & $1.06(0.95,1.18)$ \\
\hline Liver disease & $1.16(1.01,1.33)$ & $1.16(1.05,1.29)$ & $1.03(0.84,1.27)$ \\
\hline Metastatic cancer & $0.92(0.61,1.39)$ & $0.94(0.68,1.29)$ & $0.29(0.10,0.81)$ \\
\hline Any tumor & $1.01(0.87,1.17)$ & $0.93(0.84,1.03)$ & $0.98(0.79,1.22)$ \\
\hline Alcohol abuse & $0.77(0.64,0.93)$ & $0.94(0.82,1.09)$ & $0.75(0.53,1.06)$ \\
\hline Tobacco use & $1.01(0.92,1.10)$ & $1.13(1.06,1.20)$ & $1.03(0.92,1.17)$ \\
\hline Combined comorbidity score & $0.96(0.93,0.98)$ & $0.95(0.94,0.97)$ & $1.01(0.97,1.05)$ \\
\hline \multicolumn{4}{|l|}{ History of medication use in prior year } \\
\hline Non-statin lipid-lowering drugs & $1.10(0.94,1.27)$ & $1.13(1.02,1.25)$ & $1.04(0.86,1.25)$ \\
\hline Statins & $1.03(0.93,1.15)$ & $1.02(0.96,1.10)$ & $0.89(0.77,1.02)$ \\
\hline ACE inhibitors & $1.08(0.97,1.20)$ & $1.00(0.94,1.07)$ & $0.96(0.84,1.09)$ \\
\hline ARBs & $0.98(0.85,1.13)$ & $1.10(1.01,1.20)$ & $1.06(0.90,1.25)$ \\
\hline Beta blockers & $0.91(0.81,1.02)$ & $0.91(0.85,0.97)$ & $0.94(0.83,1.07)$ \\
\hline Calcium channel blockers & $0.88(0.78,0.99)$ & $0.92(0.86,0.99)$ & $0.90(0.78,1.04)$ \\
\hline Diuretics & $1.16(1.06,1.28)$ & $1.01(0.96,1.08)$ & $1.09(0.97,1.22)$ \\
\hline Insulin & $1.55(1.28,1.87)$ & $1.36(1.20,1.53)$ & $1.04(0.81,1.34)$ \\
\hline Oral hypoglycemic drugs & $1.23(1.06,1.43)$ & $1.05(0.95,1.15)$ & $0.98(0.80,1.20)$ \\
\hline Aspirins & $0.99(0.82,1.19)$ & $0.83(0.73,0.94)$ & $0.75(0.55,1.01)$ \\
\hline Coxibs & $1.46(1.32,1.61)$ & $1.30(1.23,1.38)$ & $1.11(1.00,1.23)$ \\
\hline Non-selective NSAIDs & $1.30(1.20,1.39)$ & $1.07(1.02,1.12)$ & $1.12(1.03,1.22)$ \\
\hline Opioids & $1.18(1.09,1.27)$ & $1.19(1.13,1.25)$ & $1.21(1.10,1.34)$ \\
\hline \multicolumn{4}{|l|}{ Steroid daily dosage } \\
\hline None & Reference & Reference & Reference \\
\hline Low (<5 mg/day) & $2.42(2.25,2.60)$ & $1.65(1.57,1.73)$ & $1.52(1.38,1.68)$ \\
\hline Medium (5-10 mg/day) & $3.12(2.56,3.80)$ & $1.89(1.76,2.04)$ & $1.53(1.34,1.75)$ \\
\hline High ( $\geq 10$ mg/day) & $2.61(1.91,3.57)$ & $1.72(1.55,1.89)$ & $1.81(1.51,2.15)$ \\
\hline
\end{tabular}


Table 2 Adjusted odds ratio $(95 \% \mathrm{Cl})$ of initiation or switching biologic DMARDs (Continued)

\begin{tabular}{llll}
\hline Number of non-biologic DMARDs & & Reference & Reference \\
$\quad$ None & - & $1.54(1.42,1.66)$ & $1.44(1.27,1.64)$ \\
$\quad$ One & - & $2.40(2.17,2.67)$ & $1.98(1.65,2.36)$ \\
$\quad$ More than one & - & $1.79(1.69,1.90)$ & $0.78(0.70,0.87)$ \\
Prior MTX & - & $0.57(0.54,0.61)$ & $0.75(0.65,0.86)$ \\
Prior HCQ & - & & $1.01(1.00,1.02)$ \\
Healthcare utilization in prior year & & $0.99(0.98,0.99)$ & $1.01(1.00,1.01)$ \\
Number of prescriptions & $0.97(0.97,0.98)$ & $1.00(1.00,1.01)$ & $0.98(0.92,1.05)$ \\
Number of physician visits & $1.00(1.00,1.01)$ & $1.05(1.02,1.08)$ & $1.00(0.97,1.02)$ \\
Number of hospitalizations & $1.02(0.97,1.06)$ & $0.97(0.95,0.98)$ & \\
Number of emergency room visits & $0.98(0.96,1.00)$ & & \\
\hline
\end{tabular}

Odds ratios were adjusted for demographics, data source, calendar year, comorbidities, history of medication use, and healthcare utilization in the baseline period. $R A$ rheumatoid arthritis, DMARD disease-modifying antirheumatic drug, COPD chronic obstructive pulmonary disease, ACE angiotensin-converting enzyme, $A R B$ angiotensin II receptor blocker, Coxib Cyclooxygenase inhibitor, NSAID non-selective non-steroidal anti-inflammatory drug, MTX methotrexate, HCQ hydroxychloroquine

status, we still observed a big gap by race in biologic treatment. These observed differences might be driven by differences both in accessibility to a rheumatologist and other factors, such as patients' preferences in DMARD treatment based on benefits versus risks [13].

Our study did not find significant associations between most of the comorbidities during the baseline period and initiation/switching of biologic DMARDs among all three cohorts. However, patients with underlying metastatic cancer were less likely to switch biologic DMARDs $(\mathrm{OR}=0.29,95 \% \mathrm{CI}=0.10-0.81)$. While there is limited and conflicting evidence on the risk of cancer among biologic DMARD users, possibly due to the infrequent exposure and disease outcome [14-17], our observation suggests physicians and/or patients tend to avoid treatment with a biologic agent when patients have cancer. Use of prior medication such as Coxibs, non-selective NSAIDs, opioids, and steroids,

Table 3 Race/ethnicity disparities in the use of biologic DMARDs among RA patients in Medicaid

\begin{tabular}{|c|c|c|c|c|c|}
\hline Cohort group & Race/ethnicity & Total, $n$ & $\begin{array}{l}\text { Biologic DMARD } \\
\text { initiation/switch, } n\end{array}$ & $\begin{array}{l}\text { No biologic DMARD } \\
\text { initiation/switch, } n\end{array}$ & Adjusted OR (95\% Cl) \\
\hline \multicolumn{6}{|l|}{ Odds of biologic DMARD initiation ${ }^{a}$} \\
\hline \multirow{5}{*}{$\begin{array}{l}\text { Early untreated RA, biologic- } \\
\text { DMARD-naive group }\end{array}$} & White, non-Hispanic & 23,627 & 794 & 22,833 & Ref \\
\hline & Black, non-Hispanic & 13,531 & 275 & 13,256 & $0.59(0.51,0.68)$ \\
\hline & Other non-Hispanic & 4824 & 133 & 4691 & $0.88(0.72,1.06)$ \\
\hline & Hispanic & 9875 & 403 & 9472 & $1.24(1.08,1.42)$ \\
\hline & Total & 51,857 & 1605 & 50,252 & - \\
\hline \multirow{5}{*}{$\begin{array}{l}\text { Prevalent RA, biologic-DMARD- } \\
\text { naive group }\end{array}$} & White, non-Hispanic & 25,537 & 2570 & 22,967 & Ref \\
\hline & Black, non-Hispanic & 11,171 & 759 & 10,412 & $0.71(0.61,0.74)$ \\
\hline & Other non-Hispanic & 5950 & 521 & 5429 & $0.89(0.81,0.99)$ \\
\hline & Hispanic & 10,853 & 1219 & 9634 & $1.08(0.99,1.17)$ \\
\hline & Total & 53,511 & 5069 & 48,442 & - \\
\hline \multicolumn{6}{|l|}{ Odds of biologic DMARD switch ${ }^{a}$} \\
\hline \multirow{5}{*}{$\begin{array}{l}\text { Prevalent RA, prior biologic } \\
\text { DMARD users }\end{array}$} & White, non-Hispanic & 4181 & 532 & 3649 & Ref \\
\hline & Black, non-Hispanic & 976 & 99 & 877 & $0.71(0.55,0.90)$ \\
\hline & Other non-Hispanic & 836 & 98 & 738 & $1.08(0.85,1.41)$ \\
\hline & Hispanic & 1670 & 192 & 1478 & $0.92(0.76,1.12)$ \\
\hline & Total & 7663 & 921 & 6742 & - \\
\hline
\end{tabular}


Table 4 Switching between TNF and non-TNF biologic DMARDs

\begin{tabular}{|c|c|c|c|c|c|}
\hline \multirow[t]{2}{*}{ Prior biologic } & \multirow{2}{*}{$\begin{array}{l}\text { Number (\% of all prior } \\
\text { biologic DMARDs) }\end{array}$} & \multirow{2}{*}{$\begin{array}{l}\text { Number of switches } \\
\text { (\% of each prior biologic } \\
\text { DMARD) }\end{array}$} & \multirow{2}{*}{$\begin{array}{l}\text { Adjusted }{ }^{\mathrm{a}} \mathrm{OR} \text { of } \\
\text { any switch }(95 \% \mathrm{Cl})\end{array}$} & \multicolumn{2}{|c|}{ Switch to TNF or to non-TNF inhibitors ${ }^{\mathrm{b}}$} \\
\hline & & & & $\begin{array}{l}\text { Number of switches } \\
\text { to TNF inhibitors (\%) }\end{array}$ & $\begin{array}{l}\text { Number of switches } \\
\text { to non-TNF inhibitors (\%) }\end{array}$ \\
\hline \multicolumn{6}{|l|}{ TNF inhibitors } \\
\hline Etanercept & $11,753(50.6)$ & $1223(10.4)$ & Reference & $989(80.9)$ & $234(19.1)$ \\
\hline Adalimumab & $5119(22.0)$ & $732(14.3)$ & $1.22(1.10,1.35)$ & $573(78.3)$ & $159(21.7)$ \\
\hline Certolizumab & $104(0.4)$ & $20(19.2)$ & $1.44(0.86,2.39)$ & $8(40.0)$ & $12(60.0)$ \\
\hline Golimumab & $130(0.6)$ & $35(26.9)$ & $2.24(1.48,3.37)$ & $22(62.9)$ & $13(37.1)$ \\
\hline Infliximab & $5102(22.0)$ & $567(11.1)$ & $1.06(0.95,1.19)$ & $377(66.5)$ & $190(33.5)$ \\
\hline \multicolumn{6}{|c|}{ Non-TNF inhibitor biologic agents } \\
\hline Abatacept & $407(1.8)$ & $70(17.2)$ & $1.31(0.99,1.72)$ & $40(57.1)$ & $30(42.9)$ \\
\hline Anakinra & $260(1.1)$ & $85(32.7)$ & $3.20(2.41,4.25)$ & 79 (92.9) & $6(7.1)$ \\
\hline Rituximab & $334(1.4)$ & $27(8.1)$ & $0.57(0.38,0.85)$ & $16(59.3)$ & $11(40.7)$ \\
\hline Tocilizumab & $22(0.1)$ & $2(9.1)$ & $0.59(0.14,2.60)$ & $0(0.0)$ & $2(100)$ \\
\hline Total & $23231(100)$ & $2761(11.9)$ & - & $743(76.2)$ & 657 (23.8) \\
\hline
\end{tabular}

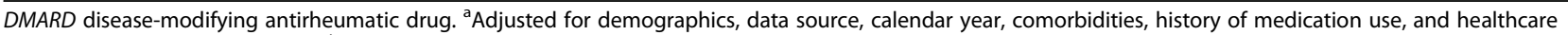
utilization in the baseline period. ${ }^{\mathrm{b} T N F}$ inhibitors include adalimumab, certolizumab, etanercept, golimumab, and infliximab; non-TNF inhibitors include abatacept, anakinra, rituximab, and tocilizumab

which are probably indicative of symptoms of active RA, were positively associated with biologic DMARD initiation/switching in patients with early or untreated RA or those with prevalent RA. It suggests that before starting biologic treatment, clinicians would start with steroid treatment for patients in the early stage of the disease. Additionally, the use of non-biologic DMARDs aligned well with ACR guideline recommendations, whereby patients who had more nonbiologic DMARDs before were more likely to initiate or switch biologic DMARDs due to non-response to the non-biologic treatment.

The most commonly used non-biologic drugs, MTX and HCQ, were negatively associated with biologic DMARD switching. Not switching to other biologic DMARDs may reflect better effectiveness or safety of current biologic treatment. In other words, it may support the commonly agreed fact among rheumatologists and in clinical trials that the effects of biologic DMARDs are greater in patients with RA when combined with MTX and/or HCQ $[2,18]$.

Etanercept, first approved by the FDA in 1988, still remains a popular biologic drug for RA treatment. It has been considered as one of the safest biologic drugs with longer treatment duration, low risk of infection, and fewer adverse events in the elderly $[15,19,20]$. Other TNF inhibitors such as adalimumab and infliximab were also used frequently with a low proportion of patients switching. Among the non-TNF biologic DMARDs, patients on anakinra had the highest odds of switching to other biologic DMARDs. It is reported in other studies that anakinra has poorer benefit and safety outcomes when compared to other biologic DMARDs [21]. Other non-TNF inhibitor biologic DMARDs, abatacept and rituximab, were unlikely to be switched when compared to etanercept. Based on 2012 ACR RA treatment recommendations, the anti-TNF biologic DMARDs, abatacept or rituximab, were recommended for initiation of a biologic DMARD after non-biologic DMARD monotherapy or combination therapy. Also, if a patient still suffered from moderate or high disease activity, then the recommendation was to change to another anti-TNF or to a non-TNF biologic DMARD [2]. Our result showed that TNF inhibitors were preferred in most circumstances, as both first-line and second-line choices. Adalimumab, etanercept, and infliximab were most common subsequent biologic DMARDs; abatacept and rituximab are used most commonly among non-TNF inhibitors as subsequent biologic DMARDs. Thus, the pattern of biologic DMARD initiation and switching in the real world setting follows the previously described ACR recommendations [2].

This study is the largest cohort study of DMARD biologic utilization patterns and trends, representing both commercially and publicly insured populations across the USA. The time span of the study covered not only the utilization of early biologic DMARDs, but also included the newer biologic DMARDs that came to the market recently. It also presented over 10 years of utilization pattern, which is not reported elsewhere in previous studies. Additionally, the United Healthcare and Medicaid databases provided comprehensive baseline characteristics of patients. 
However, there are some limitations to our study. First, we acknowledge that potential misclassification of patients in the different RA groups is possible (early untreated versus prevalent). Yet, we required all patients to have continuous insurance enrollment during the study period to avoid the misclassification due to insurance switching. In addition, patients were required not to have used any DMARDs during the baseline period to be classified as having early untreated RA. It is unlikely that a patient with prevalent RA had not used any DMARD prescriptions for more than 1 year. Thus, we believe that the likelihood of RA group misclassification is minimal. Second, although our study databases provide comprehensive patient information including comorbidity, history of medication, and healthcare utilization, they still lack some clinical details such as duration or disease activity index of RA, which may have resulted in residual confounding. To minimize such confounding, we stratified the cohort into three different disease stages based on previous RA diagnosis and DMARD prescriptions and adjusted for use of analgesics and steroids, and healthcare utilization. Third, we had a limited sample size for tocilizumab and tofacitinib users as these drugs were approved more recently. This yielded wide confidence intervals for odds ratios for switching, making it hard to make any conclusions on these biologic DMARDs. Future studies are needed on the patterns of use of these newer biologic DMARDs. Last, racial/ethnicity data were limited to the Medicaid population. Therefore, our findings on racial disparities may not be generalizable to commercially insured patients.

\section{Conclusions}

This large longitudinal cohort study of RA patients enrolled in either a public or private health plan indicated important factors that are associated with biologic DMARD utilization patterns. Notably, we demonstrated potential disparities in access to biologic DMARDs in the early phases of RA by insurance status and race. Future educational interventions aimed at addressing these disparities are warranted to ensure equitable access to biologic medications and achieve optimal disease control in RA.

\footnotetext{
Abbreviations

ACE: Angiotensin-converting enzyme; ACR: American College of Rheumatology; ARB: Angiotensin II receptor blockers; Cl: Confidence interval; COPD: Chronic obstructive pulmonary disease;

Coxibs: Cyclooxygenase inhibitors; DMARD: Disease-modifying antirheumatic drugs; FDA: Food and Drug Administration; HCQ: Hydroxychloroquine; ICD-9: International Classification of Disease, Ninth Revision; MAX: Medicaid Analytic eXtract; MTX: Methotrexate; NSAIDS: Non-selective non-steroidal anti-inflammatory drugs; OR: Odds ratio; RA: Rheumatoid arthritis; TNF: Tumor necrosis factor
}

\section{Funding}

This study was supported by an investigator-initiated grant from Pfizer, Inc. The study was conducted by the authors independent of the sponsor. The sponsor was given the opportunity to make non-binding comments on a draft of the manuscript, but the authors retained the right of publication and to determine the final wording.

\section{Availability of data and materials}

Patient level data are not available due to privacy concerns. Programming codes used for statistical analysis and study protocol are available upon request.

\section{Authors' contributions}

All authors participated in drafting or revising the article. SCK takes responsibility for the integrity of the data and the accuracy of the data analysis. Study conception and design: SCK, RJD. Analysis and interpretation of data: YJ, JL, NKC, RJD, SCK. All authors read and approved the final manuscript.

\section{Competing interests}

There are no competing interests among any of the authors. SCK receives research grants to the Brigham and Women's Hospital from Lilly, Genentech, Pfizer, Bristol-Myers Squibb, and AstraZeneca. RJD receives research grants to the Brigham and Women's Hospital from Merck.

\section{Consent for publication}

Not applicable.

\section{Ethics approval and consent to participate}

The study protocol was approved by the Institutional Review Board of the Brigham and Women's Hospital. Personal identifiers were removed from the dataset before the analysis to protect subject confidentiality. Patient informed consent was therefore not required.

\section{Publisher's Note}

Springer Nature remains neutral with regard to jurisdictional claims in published maps and institutional affiliations.

\section{Author details}

${ }^{1}$ Division of Pharmacoepidemiology and Pharmacoeconomics, Brigham and Women's Hospital, 1620 Tremont Street, Suite 3030, Boston, MA 02120, USA. ${ }^{2}$ Institute of Environmental Medicine, Medical Research Center, Seoul National University, Seoul, Republic of Korea. ${ }^{3}$ Department of Health Convergence, Ewha Womans University, Seoul, Republic of Korea. ${ }^{4}$ Division of Rheumatology, Immunology and Allergy, Brigham and Women's Hospital, Boston, MA, USA.

Received: 13 October 2016 Accepted: 9 June 2017

Published online: 05 July 2017

\section{References}

1. Helmick CG, Felson DT, Lawrence RC, Gabriel S, Hirsch R, Kwoh CK, Liang $\mathrm{MH}$, Kremers HM, Mayes MD, Merkel PA, et al. Estimates of the prevalence of arthritis and other rheumatic conditions in the United States: Part I. Arthritis Rheum. 2008;58(1):15-25.

2. Singh JA, Furst DE, Bharat A, Curtis JR, Kavanaugh AF, Kremer JM, Moreland LW, O'Dell J, Winthrop KL, Beukelman T, et al. 2012 update of the 2008 American College of Rheumatology recommendations for the use of disease-modifying antirheumatic drugs and biologic agents in the treatment of rheumatoid arthritis. Arthritis Care Res. 2012;64(5):625-39.

3. Saag KG, Teng GG, Patkar NM, Anuntiyo J, Finney C, Curtis JR, Paulus HE, Mudano A, Pisu M, Elkins-Melton M, et al. American College of Rheumatology 2008 recommendations for the use of nonbiologic and biologic disease-modifying antirheumatic drugs in rheumatoid arthritis. Arthritis Rheum. 2008;59(6):762-84.

4. Kim SC, Yelin E, Tonner C, Solomon DH. Changes in use of diseasemodifying antirheumatic drugs for rheumatoid arthritis in the United States during 1983-2009. Arthritis Care Res. 2013;65(9):1529-33.

5. DeWitt EM, Lin L, Glick HA, Anstrom KJ, Schulman KA, Reed SD. Pattern and predictors of the initiation of biologic agents for the treatment of rheumatoid arthritis in the United States: an analysis using a large observational data bank. Clin Ther. 2009;31(8):1871-58. 
6. Kim G, Barner JC, Rascati K, Richards K. Factors associated with the initiation of biologic disease-modifying antirheumatic drugs in Texas Medicaid patients with rheumatoid arthritis. J Manag Care Specialty Pharmacy. 2015;21(5):401-7.

7. Desai RJ, Rao JK, Hansen RA, Fang G, Maciejewski ML, Farley JF. Predictors of treatment initiation with tumor necrosis factor-a inhibitors in patients with rheumatoid arthritis. J Manag Care Pharm. 2014;20(11):1110-20.

8. Yelin E, Tonner C, Kim SC, Katz JN, Ayanian JZ, Brookhart MA, Solomon DH. Sociodemographic, disease, health system, and contextual factors affecting the initiation of biologic agents in rheumatoid arthritis: a longitudinal study. Arthritis Care Res. 2014;66(7):980-9.

9. Gagne JJ, Glynn RJ, Avorn J, Levin R, Schneeweiss S. A combined comorbidity score predicted mortality in elderly patients better than existing scores. J Clin Epidemiol. 2011;64(7):749-59.

10. Fischer MA, Polinski JM, Servi AD, Agnew-Blais J, Kaci L, Solomon DH. Prior authorization for biologic DMARDs: a description of United States Medicaid programs. Arthritis Rheum. 2008:59(11):1611-7.

11. Solomon DH, Ayanian JZ, Yelin E, Shaykevich T, Brookhart MA, Katz JN. Use of disease-modifying medications for rheumatoid arthritis by race and ethnicity in the National Ambulatory Medical Care Survey. Arthritis Care Res (Hoboken). 2012;64(2):184-9.

12. Constantinescu F, Goucher S, Weinstein A, Fraenkel L. Racial disparities in treatment preferences for rheumatoid arthritis. Med Care. 2009:47(3):350-5.

13. Constantinescu F, Goucher S, Weinstein A, Smith W, Fraenkel L. Understanding why rheumatoid arthritis patient treatment preferences differ by race. Arthritis Rheum. 2009;61(4):413-8.

14. Setoguchi S, Solomon DH, Weinblatt ME, Katz JN, Avorn J, Glynn RJ, Cook EF, Carney G, Schneeweiss S. Tumor necrosis factor a antagonist use and cancer in patients with rheumatoid arthritis. Arthritis Rheum. 2006;54(9): 2757-64.

15. Askling J, Fahrbach K, Nordstrom B, Ross S, Schmid CH, Symmons D. Cancer risk with tumor necrosis factor alpha (TNF) inhibitors: meta-analysis of randomized controlled trials of adalimumab, etanercept, and infliximab using patient level data. Pharmacoepidemiol Drug Saf. 2011;20(2):119-30.

16. Bongartz T, Warren FC, Mines D, Matteson EL, Abrams KR, Sutton AJ. Etanercept therapy in rheumatoid arthritis and the risk of malignancies. A systematic review and individual patient data meta-analysis of randomized controlled trials. Ann Rheum Dis. 2009;68(7):1177-83. doi:10.1136/ard.2008. 094904

17. Gossen N, Jacob L, Kostev K: Second-line therapy with biological drugs in rheumatoid arthritis patients in German rheumatologist practices: a retrospective database analysis. Rheumatol Int. 2016;36(8):1113-8. doi:10.1007/s00296-016-3448-9.

18. Weinblatt ME, Kremer JM, Bankhurst AD, Bulpitt KJ, Fleischmann RM, Fox RI, Jackson CG, Lange M, Burge DJ. A trial of etanercept, a recombinant tumor necrosis factor receptor:Fc fusion protein, in patients with rheumatoid arthritis receiving methotrexate. N Engl J Med. 1999:340(4):253-9.

19. Fleischmann R, Baumgartner SW, Weisman MH, Liu T, White B, Peloso P. Long term safety of etanercept in elderly subjects with rheumatic diseases. Ann Rheum Dis. 2006;65(3):379-84.

20. lannone F, Gremese E, Atzeni F, Biasi D, Botsios C, Cipriani P, Ferri C, Foschi $\checkmark$, Galeazzi M, Gerli R, et al. Long term retention of tumor necrosis factoralpha inhibitor therapy in a large Italian cohort of patients with rheumatoid arthritis from the GISEA registry: an appraisal of predictors. J Rheumatol. 2012;39(6):1179-84.

21. Reynolds A, Koenig AS, Bananis E, Singh A. When is switching warranted among biologic therapies in rheumatoid arthritis? Expert Rev Pharmacoecon Outcomes Res. 2012:12(3):319-33.

\section{Submit your next manuscript to BioMed Central and we will help you at every step:}

- We accept pre-submission inquiries

- Our selector tool helps you to find the most relevant journal

- We provide round the clock customer support

- Convenient online submission

- Thorough peer review

- Inclusion in PubMed and all major indexing services

- Maximum visibility for your research

Submit your manuscript at www.biomedcentral.com/submit
C) Biomed Central 